1 Clinical Operational Research Unit, University College London, UK

2 London, UK

3 Bloomsbury Institute of Intensive Care Medicine, University College London, UK

c.pagel@ucl.ac.uk

Cite this as: BMJ 2021;372:n569 http://dx.doi.org/10.1136/bmj.n569 Published: 01 March 2021

\title{
"We are setting ourselves on fire to keep everyone else warm"-what does the recovery look like for NHS staff?
}

\section{Recovery from the pandemic will be as challenging as the pandemic itself, argue Christina Pagel and Edward Palmer}

\section{Christina Pagel, ${ }^{1}$ Edward Palmer 2,3}

The number of people in UK hospitals with covid-19 is currently just under $17 \mathrm{0o0}$, having reached twice the April peak in mid-January. There have been $50 \%$ more intensive care beds in use than during the first peak. More and more stories are appearing in the media about the incredible strain that staff are under. Those stories will likely disappear once occupancy has fallen significantly, but the medium and long term impact on staff will not.

The NHS needs to plan now for how to support the wellbeing of its staff over the coming months and years. A "back to normal" approach risks the mental health of staff, staff retention, and the ability to deliver NHS services over the coming years.

Even before covid-19, NHS staff were at higher risk of mental health problems, self-harm, and death by suicide than the general population. For instance, female doctors have a suicide risk up to four times higher and nurses up to five times higher than the general population. In the 2019 NHS staff survey, over $40 \%$ reported feeling unwell as a result of work related stress over the previous year. Then the pandemic hit. The first wave put the NHS under immense strain and when the BMA surveyed intensive care unit (ICU) staff last July, they found that $45 \%$ showed signs of mental ill health, and $40 \%$ signs of post-traumatic stress disorder, more than double the percentage reported by recent combat veterans. A high proportion (one in five) of ICU nurses reported thoughts of self-harm or suicide.

Then, after a summer of working flat-out to tackle the backlog of care from the first wave, the second wave started in September. In the first wave, hospitals in England spent 40 days with an occupancy of more than 10 ooo covid-19 patients. The second wave has already seen well over 80 days with over 10000 patients in hospitals. Even with the most optimistic estimates of decline, it will take time to get down to 10 ooo covid-19 patients again. And longer still to get back to the few thousand we had at the beginning of September. If restrictions are eased too quickly as the most vulnerable groups are vaccinated, a third wave of admissions among younger patients is possible in the spring. What is certain is that by the time this is over, staff will have been working beyond their limits for many months. As one doctor put it, "We are setting ourselves on fire to keep everyone else warm."

Much of the impact will be the mental trauma experienced by healthcare workers caring for patients in both far greater number and with illness severity than normal. Many patients will have died, often away from their loved ones. Many healthcare staff will have been patients themselves or have seen their colleagues become patients. Most will know of someone who was critically ill or died. An estimated $5-10 \%$ of healthcare staff will be left struggling with long covid.

These difficulties will be amplified for the thousands of healthcare workers deployed away from their specialty area and away from their team mates. This brings the added stress of feeling deskilled and out of one's depth. The feeling of working far out of your comfort zone can be a dragging stress-particularly if continued for weeks on end. This burden will be particularly acute for those who are not used to seeing death in their usual role. Added to this is the knowledge that many of your "usual" patients are not getting the benefit of your expertise for weeks and months. Will delays to their treatment have repercussions for their outcomes? How will you see them all?

Then there is the additional burden of losing months of training for junior staff, who will also often be the ones who have been providing patient facing care. Exams have been postponed, and placements-where they were meant to learn the intricacies of their craft-spent deployed on covid wards. Many will put enormous pressure on themselves to try to catch up as soon as possible, leading to further strain.

Staff are physically and mentally exhausted. As they manage the current peak they will be in coping mode. It is only when the immediate pressure abates that staff will have time to process what has happened and will need to access support. But by summer the pressure will be on the NHS, not just to deliver "normal" levels of care to all who need it, but to offer extra clinics, extra surgeries, extra everything to start tackling the backlog of cases that will take years to work through. Trying to do this without letting traumatised staff recover or putting in place active measures to support their wellbeing would be an enormous mistake. It is imperative that staff have access to evidence based psychological therapies in the recovery period in order to prevent poor mental health outcomes.

The NHS has already increased staff access to wellbeing support through counselling or advice on wellbeing. However, access can vary greatly across different NHS trusts. All of this must continue and be expanded as pressure eases. Leadership at all levels must encourage staff to care for themselves and each other, openly discussing impacts on them to reduce stigma and encourage conversation and mutual support. Careful plans must be made to allow all staff 
to access evidence based psychological support as part of their work hours. They must be enabled and encouraged to take the annual leave they have sacrificed, in long enough blocks to allow for recuperation. This might mean less activity in the short term, but it will mean more activity in the longer term as staff recover and recharge.

Perhaps most importantly, gaps in NHS staffing, particularly for nurses and doctors, must be urgently tackled through maximising staff retention, with better rates of pay, encouragement of immigration, and, for the longer term, greatly increased training places with proper funding, particularly for nursing qualifications.

Recovery from the pandemic will be as challenging as the pandemic itself. Maintaining the health and wellbeing of our staff-and resuming the delivery of high quality care for all-will require courageous and novel thinking. Staff wellbeing and patient care are intrinsically interdependent. To ignore the matter is to invite further catastrophe into an already fragile system.

Competing interests: None declared. 